\title{
33. STRUCTURE AND DYNAMICS OF THE GALACTIC SYSTEM
}

\author{
PRESIDENT: M. Mayor (Switzerland) \\ VICE-PRESIDENT: Leo Blitz (USA)
}

\section{BUSINESS MEETING}

\section{IAU report on astronomy, symposia etc.}

For scveral triennia commission 33 had maintained the tradition to publish a "long report" in addition to the IAU report published in Transactions A. In view of the now existing excellent publication of Astronomy and Astrophysics Abstracts it appears that the 35 pages or more of the IAU report are sufficient to reasonably cover the most important progresses done in the field of Galactic Structure. Therefore the "long report" of commission 33 has been suppressed.

Jommission 33 has cosponsored the following IAU symposia or colloquia:

IAU symposium 139 "Galactic and extragalactic background radiation" held in Heidelberg (June 1989); IAU symposium 144 "The interstellar disk-halo connection in galaxy" held in Leiden (June 1990); IAU symposium 148 "The Magellanic Clouds and their dynamical interaction with the Milky Way" held in Sydney (July 1990); IAU symposium 149 "The stellar populations of galaxies" held in Angra dos Reis (August 1991); IAU symposium 150 "The astrochemistry of cosmic phenomena" held in Campos de Jordao (August 1991); IAU symposium 153 "Galactic bulges" to be held in Ghent in August 1992; IAU symposium 156 "Developments of astrometry and their impacts on astrophysics and geodynamics" to be held in Shanghai in September 1992; IAU symposium 155 "Planetary nebulae" to be held in Innsbruck in July 1992; IAU colloquium 127 "Reference systems" held in Virginia Beach (Oct. 1990); and IAU colloquium 132 "Instability, chaos, predictability in celestial mechanics and stellar dynamics" held in Dellii (Oct. 1990).

\section{Membership}

Commission 33 approved and welcomed new members proposed by the various national committees as well as several IAU members who requested to join the commission. These included:

Aguilar Chin Luis, USA; Bienaymé Olivier, France; Chapman Jessica, Australia; Cubarsi Rafael, Spain; Fujiwara Takao, Japan; Gemmo Alessandra, Italy; Gupta Sunil, India; Hakkila Jon Eric, USA; Hanami Hitoshi, Japan; Lee Hyung Mok, Korea; Leisawitz David, USA; Polyachenko Valerij, USSR; Polymilis Chronis, Greece; Raharto Moedj, Indonesia; Robin Annie, France; Ruelas-Mayorga R, Mexico; Sanz I Subirana Joanne, Spain; Schechter Paul, USA; Seimenis Joln, Greece; Spergel David, USA; Sygnet Jean-François, France; Wyse Rosemary, USA; Zachilas Loukas, Greece;

as wcll as:

Aizu Ko, Japan; Andersen Jolanmes, Denmark; Banlatti D., India; Dejonghe Herwig, Belgium; Fridmanm Aleksey, USSR; Gottesman Stephen, USA; Grenon Michel, Switzerland; Latham David, USA; Liebert James, USA; Lu Philip, USA; Matteucci Francesca, Italy; Murray Andrew, UK; Nordstroem Birgitta, Denmark; Norman Colin, USA; Oblak Edonard, France; Pandey A.K., India; Pavlovskaya E., USSR; Rebeirot Edith, France; Sargent Anneila, USA; Sobouti Yousef, Iran; Valtonen Mauri, Finland; Whitelock Patricia, Australia; Xiang Delin, China. 
We regret the death of our colleagues Prof. Jöran Ramberg from Uppsala and Kyrill Ogorodnikov from Leningrad.

\section{Officers}

The new President of commission 33 is Leo Blitz of USA. James Binney from U.K. is the new VicePresident. The members of the Organizing Committee for the upcoming triennium 1991-1994 are Michel Mayor, Switzerland (past President), Catherine Cesarsky, France, Gerard Gilmore, UK, and the new members: Francesca Matteucci, Germany, Yuzuru Yoshii, Japan, Aleksey Fridmann, USSR, Agris Kalnajs, Australia, Johannes Bloemen, Netherlands and Mark Morris, USA.

IV. Working Group on "Galactic Constants"

F.J. Kerr: "Galactic Constants"

J.F. Kerr presented a short report on recent determinations of the Galactic constants $\mathrm{R}_{O}$ and $\Theta_{O}$, on behalf of the Commission's Working Group on Galactic Constants. This covered a period of six years since the last major report on this subject. The unweighted mean of ten determinations of $\mathrm{R}_{O}$ came to $7.8 \pm 0.4 \mathrm{kpc}$, and the mean of four determinations of the circular velocity was $216 \mathrm{kms}^{-1}$. Neithcr of these is sufficiently different from the currently-accepted values to lead to any recommendation for change.

The Working Group was terminated at this point, with thanks. Kerr was asked to continue to monitor the situation for future developments, including also the constants A and B. Any new information on any of the four constants should be sent to him.

In the very near future the space astrometric mission HIPPARCOS with its large number of high precision stellar proper motions will probably allow to fully reconsider the domain of galactic constants. Michel Crézé is invited to establish the contact between our commission and the HIPPARCOS team and, if necessary, to report at the next Goneral Assembly.

\section{SCIENTIFIC MEETINGS}

At the General Assembly Commission 33 was among the cosponsoring commissions of three Joint Discussions: JDII "Reference Systems" chaired by J.A. Hughes; JDV "Origin of Stars and Planetary Systems" chaired by André Brahic and JDVI "HIPPARCOS - An Assessment" chaired by Catherine Turon.

"Impact of radial velocity observations on Galactic Structure and Evolution"

Chaired by David Latham and Michel Mayor

The speakers were Leo Blitz (Maryland) reviewing works on the shape of the galactic disk; David Latham (CfA) described the kinematics of the thick disk; Michel Grenon (Geneva Observatory) reported on connections between disk, halo and bulge and finally Dante Minniti (Arizona) presented bulge kinematics.

\section{"Large Scale Distribution of Gas and Stars in the Milky Way"}

\section{Chaired by Leo Blitz}

It was a double session lasting from 9.00 to 12.30. The speakers were David Spergel (Princeton), Michael Rich (Columbia), Patricia Whitelock (SAAO), David Latham (CfA), Harvey Liszt (NRAO), Leonardo Bronfman (Clile) and Tetsuo Hasegawa (Tokyo). The session was divided into two parts. The first dealt with the bulge of the Galaxy, and the second with the disk and halo. The session on the bulge featured direct new evidence that the bulge of the Galaxy is actually a bar, and an independent confirmation of this picture from IRAS observations of Mira variables. An important study of the metallicity of the bulge shows that the stars have evolved as if they were in a closed box without significant mixing of the inner disk population. The work on the disk and the halo included a talk on the evidence for metallicity differences between the thin and thick disk populations. Papers on the gas discussed an overview of the distribution of molecular gas as well as new evidence that Giant Molecular Clouds become less dense with increasing distance to the galactic center. Work was also presented describing outstanding anomalies in our understanding of the distribution of atomic gas. The papers will be combined with two papers from the session on the galactic center organized by D.Y. Gezari (GSFC), and published by Kluwer. 
"The Galactic Nucleus"

Chaired by Dan Gezari

This session was held jointly with and immediately following the Commission 40 session "The Galactic Center Region and Pulsars" (Chairman: Peter Mezger). D. Gezari presented extensive infrared eightcolor $(5-18 \mu \mathrm{m})$ imaging array observations of the Sgr A West complex with his Goddard 58x62 pixel Array Camera. Detailed modelling of the dust density and temperature structure of the region suggest that the brightest compact IRS sources are intermally heated. This result as well as a correlation found between temperature enhancements on the extended ridge and newly discovered HeI emission line stars imaged by Genzel et al. indicate that imbedded sources distributed through the ridge could provide the luminosity observed in the Galactic Center, and that an exotic central engine or black hole at Sgr $A^{*}$ need not be invoked. D. Aitken described the first polarimetry with an array detector, using the Goddard infrared camera at $12.4 \mu \mathrm{m}$, of the central parsec of the Sgr A West complex. Diffraction-limited sampling revealed smooth magnetic field structure tlurough the emission ridge made up of the northern arm and east-west bar, unperturbed by the cluster of sources at IRS16. This implies that the IRS16 sources must be out of the plane of the ridge system. This field structure is modeled by an elliptical flow pattern of material with one focus at Sgr $A^{*}$. P. Mezger described new constraints on the spectrum of Sgr $A^{*}$ over a wide range of radio, sub-millimeter and infrared wavelengths. The revised spectrum was modeled as emission from a $500 \mathrm{M}_{\odot}$ dust cloud which becomes optically thick at $1-\mathrm{mm}$. H. Zinnecker reported new $1 \mu \mathrm{m}$ CCD images of the Galactic Center showing astrometry of several new near-infrared point sources near Sgr A*, including candidates for stellar objects at the Galactic Center, but no definitive identification with Sgr A* could be made. J. Pipher discussed extensive infrared Brackett line imaging observations and summarized detection of point-like sources at the Galactic Center which have no radio counterparts, possibly Wolf-Rayct stars, and measured velocities indicative of stellar winds. Lunar occultations also revealed double sources at IRS1 and IRS13. F. Yusef-Zadeh described high resolution VLA observations at 2-cm with 0.3 arcsec resolution which showed bow-shock structure associated with the mass-loosing envelope of the supergiant star IRS7 lying 1 light year in projection from the Galactic Center (IRS16/Sgr $A^{*}$ ). This bow shock structure was modelled in terms of two colliding winds, one from the IRS16 region and the other from IRS7. In addition, a tail of ionized gas on IRS7 was detected trailing directly away from the Galactic Center, seen as further evidence of this complex interaction. 